\title{
SPICAM observations and modeling of Mars aurorae
}

\author{
Lauriane Soret $^{\mathrm{a}, *}$, Jean-Claude Gérard ${ }^{\mathrm{a}}$, Ludivine Libert $^{\mathrm{a}}$, Valery I. Shematovich ${ }^{\mathrm{b}}$, Dmitry V. Bisikalo ${ }^{\mathrm{b}}$, \\ Arnaud Stiepen ${ }^{a}$, Jean-Loup Bertaux ${ }^{c}$
}

a Laboratoire de Physique Atmosphérique et Planétaire, Université de Liège, Liège, Belgium

${ }^{\mathrm{b}}$ Institute of Astronomy, Russian Academy of Sciences, Moscow, Russia

${ }^{c}$ LATMOS, Université de Versailles Saint-Quentin-en-Yvelynes, Guyancourt, France

\section{A R T I C L E I N F O}

\section{Article history:}

Received 1 April 2015

Revised 7 September 2015

Accepted 16 September 2015

Available online 26 September 2015

\section{Keywords:}

Mars, atmosphere

Aurorae

Ultraviolet observations

Terrestrial planets

\begin{abstract}
A B S T R A C T
Martian aurorae have been detected with the SPICAM instrument on board Mars Express both in the nadir and the limb viewing modes. In this study, we focus on three limb observations to determine both the altitudes and the intensities of the auroral emissions. The $\mathrm{CO}\left(\mathrm{a}^{3} \Pi-\mathrm{X}^{1} \Sigma\right)$ Cameron bands between 190 and $270 \mathrm{~nm}$, the CO $\left(\mathrm{A}^{1} \Pi-\mathrm{X}^{1} \Sigma^{+}\right)$Fourth Positive system (CO 4P) between 135 and $170 \mathrm{~nm}$, the $\mathrm{CO}_{2}^{+}$ $\left(\mathrm{B}^{2} \Sigma_{\mathrm{u}}^{+}-\mathrm{X}^{2} \Pi_{\mathrm{g}}\right)$ doublet at $289 \mathrm{~nm}$, the $\mathrm{OI}$ at $297.2 \mathrm{~nm}$ and the $130.4 \mathrm{~nm}$ OI triplet emissions have been identified in the spectra and in the time variations of the signals. The intensities of these auroral emissions have been quantified and the altitude of the strongest emission of the CO Cameron bands has been estimated to be $137 \pm 27 \mathrm{~km}$. The locations of these auroral events have also been determined and correspond to the statistical boundary of open-closed magnetic field lines, in cusp-like structures. The observed altitudes of the auroral emissions are reproduced by a Monte-Carlo model of electron transport in the Martian thermosphere for mono-energetic electrons between 40 and $200 \mathrm{eV}$.

No correlation between electron fluxes measured in the upper thermosphere and nadir auroral intensity has been found. Here, we simulate auroral emissions observed both at the limb and at the nadir using electron energy spectra simultaneously measured with the ASPERA-3/ELS instrument. The simulated altitudes are in very good agreement with the observations. We find that predicted vertically integrated intensities for the various auroral emissions are overestimated, probably as a consequence of the inclination and curvature of the magnetic field line threading the aurora. However, the relative brightness of the $\mathrm{CO}$ and $\mathrm{CO}_{2}^{+}$emissions is in good agreement with the observations.
\end{abstract}

(c) 2015 Elsevier Inc. All rights reserved.

\section{Introduction}

Even if the presence of aurorae in the Mars nightside atmosphere was expected (Fox, 1992), auroral emissions have only been detected by Bertaux et al. (2005a) with the SPICAM (Spectroscopy for the Investigation of the Characteristics of the Atmosphere of Mars) UV instrument on board Mars Express, which was launched in 2003. They observed a strong emission peak lasting $7 \mathrm{~s}$ during orbit 716, with a spectral composition quite different from the NO nightglow emission (Bertaux et al., 2005b; Cox et al., 2008). In the auroral spectrum, the main emissions are the $\mathrm{CO}$ $\left(\mathrm{a}^{3} \Pi-\mathrm{X}^{1} \Sigma\right)$ Cameron bands between 180 and $240 \mathrm{~nm}$, the CO $\left(\mathrm{A}^{1} \Pi-\mathrm{X}^{1} \Sigma^{+}\right)$(CO 4P) Fourth Positive system between 135 and $170 \mathrm{~nm}$, the $\mathrm{CO}_{2}^{+}\left(\mathrm{B}^{2} \Sigma_{\mathrm{u}}^{+}-\mathrm{X}^{2} \Pi_{\mathrm{g}}\right)$ doublet at $288.3-289.6 \mathrm{~nm}$ and the OI emission at $297.2 \mathrm{~nm}$. They also pointed out that a close correlation between the location of this observed emission and

\footnotetext{
* Corresponding author.
}

the position of the crustal field anomalies (cusp-type regions). They deduced that the auroral emissions are caused by energetic electron fluxes moving along the crustal magnetic field lines and exciting the upper atmosphere of Mars.

Leblanc et al. (2008) further searched for auroral signatures using the limb and nadir viewing modes of the SPICAM UV instrument. They found nine additional detections on the Mars nightside: one at the limb and eight in the nadir direction. These auroral emissions were observed in six orbits spread over the database, some of them showing several events a few minutes apart. They could quantify the CO Cameron bands emission and, in some cases, the $\mathrm{CO}_{2}^{+}$doublet emission. The locations of these aurorae relative to the statistical map of open/closed magnetic field lines from the Mars Global Surveyor (MGS) measurements of Brain et al. (2007) seem to confirm that aurorae occur in the presence of cusp-like magnetic field line structures. They also compared these detections with simultaneous ASPERA-3 particle measurements and MARSIS total electron content measurements. Although dependence seems to exist between the aurorae and the 
occurrence of energetic precipitating electrons, there is a lack of correlation between the intensity of the incident electron flux and the total electron content or the auroral emission intensities.

Gérard et al. (2015) reexamined a larger SPICAM database and detected additional UV aurora during nadir observations. They confirmed that Mars aurorae are located near the open-closed magnetic field line boundary in cusp-like structures and evaluated the brightness of the $\mathrm{CO}$ Cameron bands and $\mathrm{CO}_{2}^{+}$emissions. They also compared the UV auroral detections with concurrent ASPERA3 measurements and determined the characteristics of the electron energy spectra at the time of the most likely associated electron flux enhancements but did not find a clear quantitative correlation between the observed brightness and the precipitated energy flux.

In this study, we focus on limb observations, which will be presented in Section 2. While two detections during orbit 716 and one during orbit 2800 were already known (Bertaux et al., 2005a; Leblanc et al., 2008), we detected an additional auroral signature during orbit 2800 . Results derived from these observations, such as the altitude of the emissions and the duration of the aurorae will be presented. The intensities of the CO Cameron bands and the $\mathrm{CO}_{2}^{+}$ emissions are quantified, as well as those of the CO 4P bands and the $130.4 \mathrm{~nm}$ OI emissions. These observations are then compared with numerical simulations based on the model of Shematovich et al. (1994, 2008). It is an electron transport model based on a direct simulation Monte Carlo method in the Martian atmosphere that will be described in more detail in Section 3. Simulations are made for mono-energetic electrons and are compared to the auroral observations. The Monte Carlo model has also been modified so that ASPERA energetic spectra can be used as input parameters of the model. The altitudes of the aurorae are calculated as well as the intensities of the emissions forming the auroral signature.

\section{Auroral detections at the limb}

\subsection{SPICAM observations}

Auroral emissions have been detected with the SPICAM UV instrument on board the ESA Mars Express spacecraft, which is in a quasi polar orbit. The SPICAM UV spectrograph covers a 118-305 nm wavelength range. Every second, it acquires five spectra recorded in five adjacent fields of view (spatial bins), each extending along $0.32^{\circ}$ in the sky. Two of these spatial bins ( 1 and 2 ) provide the best spectral resolution $(\sim 1.5 \mathrm{~nm})$ but a lower photometric sensitivity, and two others ( 4 and 5 ) have a lower spectral resolution ( $\sim 6 \mathrm{~nm}$ ) but an 8 times higher sensitivity. The third spatial bin will not be used for quantitative study here because its sensitivity is intermediate and has not been properly calibrated (Leblanc et al., 2008). Further details about the SPICAM instrument are given by Bertaux et al. (2006) and Leblanc et al. (2006a). SPICAM UV can operate in various modes. Nadir observations of aurorae have recently been analyzed in detail by Gérard et al. (2015). They quantified the intensities of the CO Cameron bands and the $\mathrm{CO}_{2}^{+}$doublet emissions, compared auroral events to concurrent electron precipitations and confirmed that aurorae occur near open-closed field line boundaries, in the Southern hemisphere between $150^{\circ}$ and $225^{\circ}$ of longitude. In this observational section, only the limb viewing mode has been used, so that we can also determine at what altitude aurorae occur. The line of sight of SPICAM is perpendicular to the velocity vector at pericenter. The limb profile database was searched and the following criteria for a possible auroral detection were imposed: (i) the solar zenith angle has to be greater than $100^{\circ}$ to ensure that the atmosphere is in darkness; (ii) the spacecraft must be less than $1000 \mathrm{~km}$ away from the planet, which is the altitude limit imposed by the sensitivity of the instrument to detect faint and localized emissions (Leblanc et al., 2008); (iii) observation needs to be made in the Southern hemisphere, where the magnetic field anomalies are more intense (Connerney et al., 2001). Only 30 out of 1404 limb observations acquired between 29 January 2004 and 29 March 2014 match these criteria. They have thus been individually checked and the tempo-images have been visually examined to confirm a suspected presence of an auroral emission, as shown in Fig. 1a of Bertaux et al. (2005a), by a short increase of the signal of the CO Cameron bands between the 190 and $300 \mathrm{~nm}$. Finally, the spectral composition was analyzed. To do so, spectra have been summed over the elapsed time of the signal increase and compared to a pure NO nightglow spectrum and to a confirmed auroral case (orbit 716 from Bertaux et al., 2005a), which does not contain any appreciable NO airglow contribution. This methodology has been extensively described by Gérard et al. (2015) for the nadir observations. Finally, we only retain three auroral signatures. Two of them were previously identified: one during orbit 716 (Bertaux et al., 2005a) and one during orbit 2800 (Leblanc et al., 2008). The third auroral event added to this list also appears during orbit 2800, about 2 min after the first one. Hereafter, we will refer them as Detection I, Detection II and Detection III. These auroral events and the time of their detections are listed in Table 1. Fig. 1a shows the time evolution of the signal for Detection II in the five usable spatial bins. In order to extract unambiguous quantitative parameters from these

Table 1

Auroral limb detections characteristics.

\begin{tabular}{|c|c|c|c|c|c|c|c|c|c|}
\hline \multirow[t]{2}{*}{ Orbit } & \multirow[t]{2}{*}{ Spatial bin } & \multirow[t]{2}{*}{ Time (s) } & \multirow[t]{2}{*}{ Position $A_{1}^{\mathrm{a}}(\mathrm{s})$} & \multirow[t]{2}{*}{ Peak delay (s) } & \multicolumn{2}{|c|}{ Duration (s) } & \multirow[t]{2}{*}{ Intensity $A_{0}{ }^{\mathrm{a}}(\mathrm{R})$} & \multicolumn{2}{|c|}{ Altitude (km) } \\
\hline & & & & & & Mean & & Apparent & Corrected \\
\hline \multirow[t]{5}{*}{ 716A01 } & 1 & $535-540$ & 537.2 & \multirow[t]{5}{*}{2.2} & 9 & \multirow[t]{5}{*}{9} & 4959 & 15.7 & \multirow[t]{5}{*}{$132 \pm 31$} \\
\hline & 2 & $532-540$ & 536.6 & & 9 & & 4871 & 15.5 & \\
\hline & 3 & $532-539$ & 536.2 & & 9 & & - & 15.2 & \\
\hline & 4 & $532-538$ & 535.7 & & 9 & & 4462 & 14.9 & \\
\hline & 5 & $531-538$ & 535.0 & & 9 & & 4043 & 14.7 & \\
\hline \multirow[t]{10}{*}{ 2800A02 } & 1 & $370-395$ & 381.6 & \multirow[t]{5}{*}{3.2} & 29 & \multirow[t]{5}{*}{29} & 1338 & 38.0 & \multirow[t]{5}{*}{$143 \pm 23$} \\
\hline & 2 & $370-395$ & 382.4 & & 30 & & 2371 & 47.5 & \\
\hline & 3 & $372-390$ & 382.6 & & 27 & & - & 54.5 & \\
\hline & 4 & $373-392$ & 382.7 & & 29 & & 2216 & 62.9 & \\
\hline & 5 & $373-393$ & 384.8 & & 30 & & 2396 & 70.1 & \\
\hline & 1 & $515-550$ & 519.5 & \multirow[t]{5}{*}{2.5} & 38 & \multirow[t]{5}{*}{37} & 1008 & 5.3 & \multirow[t]{5}{*}{$137 \pm 26$} \\
\hline & 2 & 509-519 & 520.6 & & 28 & & 1211 & 14.4 & \\
\hline & 3 & $515-550$ & 521.5 & & 36 & & - & 22.6 & \\
\hline & 4 & $515-550$ & 521.8 & & 40 & & 694 & 30.8 & \\
\hline & 5 & $515-550$ & 522.0 & & 42 & & 733 & 39.3 & \\
\hline
\end{tabular}

\footnotetext{
${ }^{\text {a }}$ Refers to the parameters of Eq. (1).
} 

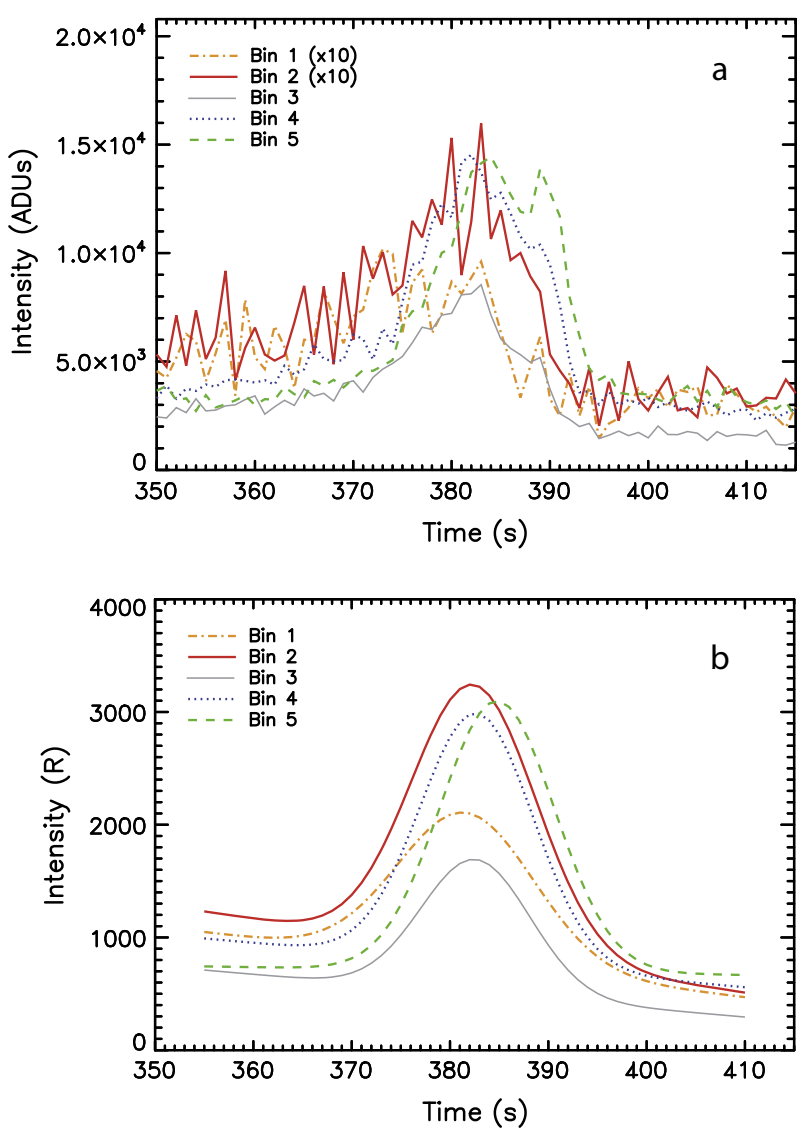

Fig. 1. (a) Time variation of the limb intensity (arbitrary units) between 190 and $300 \mathrm{~nm}$ during Detection II in the five spatial bins. (b) Combination of a Gaussian and a linear function determined with a Levenberg-Marquardt least-squares minimization procedure to reproduce the calibrated CO Cameron bands emission of Detection II between 190 and $270 \mathrm{~nm}$. The dashed-dotted lines represent the signals in spatial bins 1 and 2 . The dotted and dashed curves represent the intensities in spatial bins 4 and 5, respectively. Spatial bin 3 is not suitable for quantitative study, but confirms the presence of the signal increase.

detections, the data have been calibrated and fitted. The absolute calibration of the instrument is based upon observations of hot stars with ultraviolet fluxes previously measured in absolute units by the International Ultraviolet Explorer (IUE) and the Hubble Space Telescope (Bertaux et al., 2006). The subtraction of the offset and the non-uniform background counts was described by Leblanc et al. (2006a).

\subsection{Auroral intensity of the CO Cameron bands}

Calibrated intensity time variations have then been summed over 190 and $270 \mathrm{~nm}$ for the CO Cameron bands and individually fitted for each spatial bin. A combination of a Gaussian and a linear function $f$ was used to find the best fit with a Levenberg-Marquardt least-squares minimization procedure:

$f(t)=A_{0} \exp \left(-\frac{1}{2}\left(\frac{t-A_{1}}{A_{2}}\right)^{2}\right)+A_{3}+A_{4} t$

where $t$ is the time, $A_{0}$ is the height of the Gaussian, $A_{1}$ is the center of the Gaussian, $A_{2}$ is the width of the Gaussian, $A_{3}$ is the constant term and $A_{4}$ is the linear term. The Gaussian term actually represents the auroral emission intensity, while the linear terms account for any residual contribution from the detector, the electronics, the stray light or the underlying nitric oxide nightglow emission intensity. Auroral emissions have only been quantified when the $A_{0}$ parameter was at least a factor of two higher than the standard deviation of the background signal. Fig. $1 \mathrm{~b}$ shows the curves fitting the calibrated CO Cameron bands time evolution of Detection II in the five spatial bins. It becomes obvious that the aurora is not detected at the same time by the five spatial bins: a small delay is observed between the peak maxima. This can be explained by the geometry of limb SPICAM observations. Indeed, the five spatial bins are spatially aligned and, due to their $0.32^{\circ}$ angular size, the field of view of each spatial bin is slightly shifted. Because the spacecraft is moving, the first spatial bin will observe the aurora before the second and so on. The cartoon in Fig. 2a illustrates this situation. The delay between the first and the last maximum is estimated using the five $A_{1}$ parameters retrieved from the individual fits. Here, we find a time delay of $3.2 \mathrm{~s}$ between $381.6 \mathrm{~s}$ for the first peak of Detection II and $384.8 \mathrm{~s}$ for the last one. The time delays of Detection I and Detection III are 2.2 and $2.5 \mathrm{~s}$, respectively. They are listed in Table 1 . The duration of the aurora can also be estimated for each spatial bin from the base of the Gaussian. Table 1 indicates that Detections I, II and III respectively last 9, 29 and 37 s. Finally, the $A_{0}$ parameter gives the intensity of the CO Cameron bands emission at the maximum of the aurora for bins $1,2,4$ and 5 (due to its ambiguous calibration, bin 3 is not used to derive intensities). In average, we find intensities of $4585 \mathrm{R}$ (1 Rayleigh $=10^{6}$ photons $\mathrm{cm}^{-2} \mathrm{~s}^{-1}$ emitted in $4 \pi$ steradians), $2080 \mathrm{R}$ and $910 \mathrm{R}$ for the three auroral emissions. Intensities are thus highly variable as they can change by a factor of 5 from one observation to another. Bertaux et al. (2005a) found a CO Cameron bands intensity of $700 \pm 50 \mathrm{R}$ for
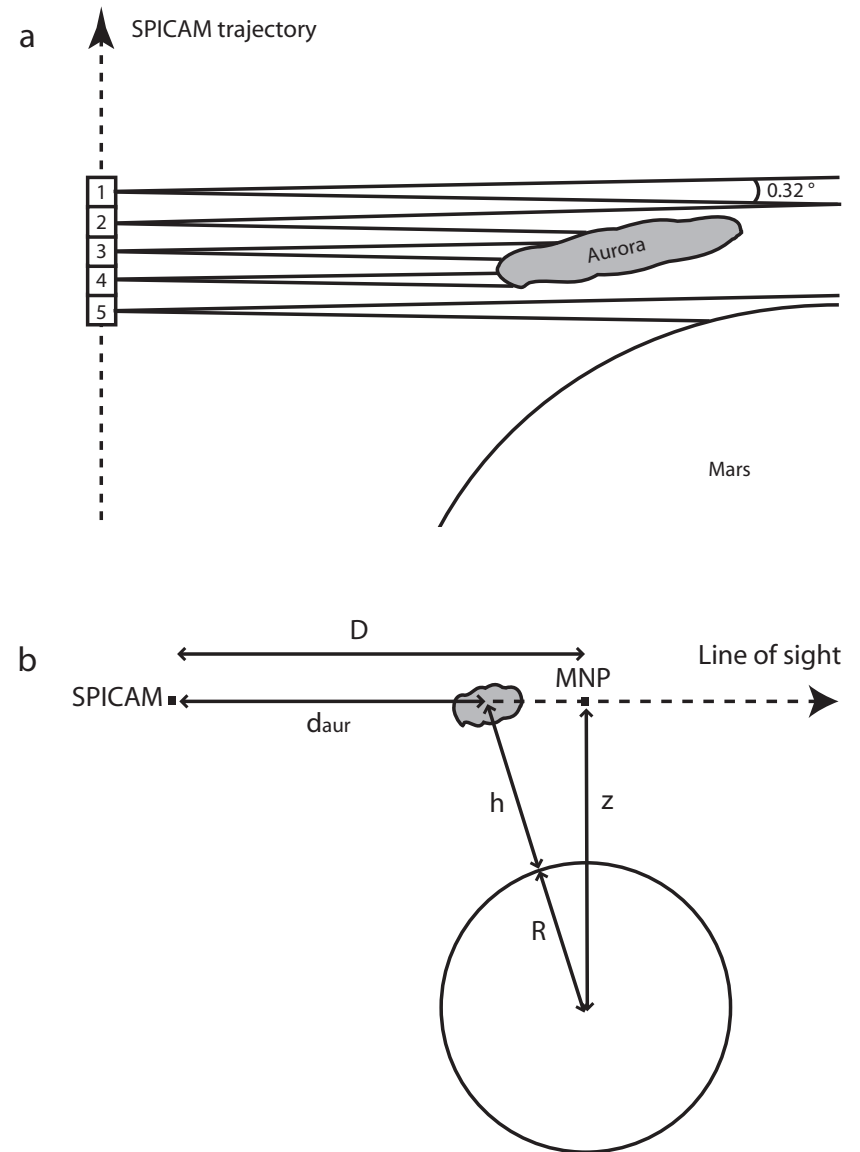

Fig. 2. (a) The five spatial bins of SPICAM UV do not detect the aurora at the same time during a limb observation: bin 1 has already observed the aurora while bins 2 , 3 and 4 are acquiring the emission signal and bin 5 has not yet started detecting it (b) The apparent altitude of the aurora $(z-R)$ can be corrected $(h)$ based on the geometry of the observation. 
Detection I but, as the calibration improved, Leblanc et al. (2006a) revised this value upward. They finally calculated a mean value over the five spatial bins of $2050 \mathrm{R}$. This is the mean intensity of the aurora during the whole duration of the emission, while we only consider the emission intensity at its maximum. They are thus consistent with each other. Leblanc et al. (2008) also studied Detection II. They found an averaged intensity of $712 \mathrm{R}$ for the emission of the CO Cameron bands. As a comparison, Leblanc et al. (2008) and Gérard et al. (2015) analyzed nadir observations of aurorae. Leblanc et al. (2008) measured mean intensities of $325 \mathrm{R}$. These nadir values are weaker than those obtained at limb since the intensities acquired in the limb mode are integrated along the line of sight. Gérard et al. (2015) found that the maximum CO Cameron bands intensity at nadir can varies from $210 \mathrm{R}$ to $2995 \mathrm{R}$.

\subsection{Altitude of the auroral emission}

A SPICAM UV limb observation actually consists in observing through the atmosphere first during a decreasing phase of the altitude of the tangent point (Mars Nearest Point - MNP), and then during its rise. Thus, the altitude range of a limb observation is scanned twice. The limb profiles were acquired around the detection time of the aurorae, between 190 and $300 \mathrm{~nm}$. A sudden increase of the intensity in the limb profiles was indeed detected. Such a profile is shown in Fig. 3. The apparent altitude of the auroral peak is readily seen. The apparent altitudes of the aurorae for each spatial bin are reported in Table 1 . They vary from 5 to $70 \mathrm{~km}$, depending on the detection and the spatial bin. As for the temporal delay between the peak intensity observed in Fig. 1, the altitude difference retrieved between the spatial bins of a given auroral detection is due to the geometry of the observation, as previously shown in Fig. 2a. These apparent peak altitudes actually represent the altitude of the tangent point of the limb line of sight at the time of the crossing of the aurora, but do not reflect the real altitude of the auroral emission itself. To estimate the actual altitude of the UV aurora, the geometry of the observation has to be taken into account. This method of deducing the peak altitude of the emission was described by Bertaux et al. (2005a), and utilizes the observed time delay between the spatial bins. First, using the spacecraft coordinates between the beginning and the end of a detection, the horizontal velocity of the instrument $v$ can be calculated. Then, by dividing the total field of view of the five spatial bins by the time delay between the temporal peaks estimated in Section 2.2 , the apparent angular velocity $\omega$ can be calculated.

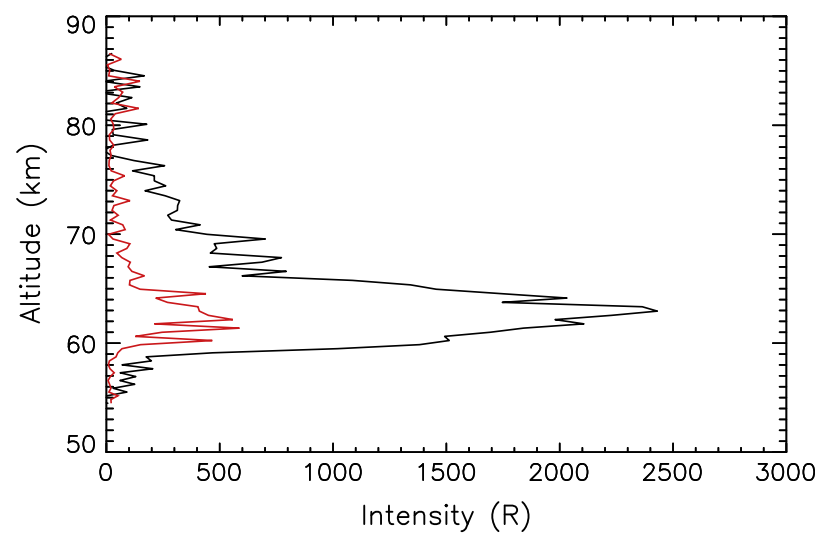

Fig. 3. Limb profile acquired during the auroral detection in spatial bin 4 along orbit 2800 between 370 and 395 s (Detection II). The black line represents the CO Cameron bands intensity, which has been spectrally integrated between 190 and $270 \mathrm{~nm}$. The red line represents the $\mathrm{CO}_{2}^{+}$intensity, spectrally integrated between 286 and $293 \mathrm{~nm}$. (For interpretation of the references to color in this figure legend, the reader is referred to the web version of this article.)
Therefore, the distance between the spacecraft and the aurora is $d_{a u r}=v / \omega$. According to Fig. $2 \mathrm{~b}$, the actual altitude of the aurora $h$ is given by:

$h=\sqrt{z^{2}+\left|D-d_{a u r}\right|^{2}}-R$

where $z$ is the distance from the MNP to the center of the planet, $D$ is the known distance of the spacecraft to the MNP and $R$ is the Martian radius. The corrected values of the auroral emissions altitudes are indicated in the last column of Table 1. Applying this method, we find that the altitude of Detection I is $132 \mathrm{~km}$, as was previously estimated by Bertaux et al. (2005a). The altitudes of Detection II and Detection III are respectively 143 and $137 \mathrm{~km}$, quite close to that of Detection I. The uncertainty on the duration of the aurora and of the time delay between the spatial bins detection is estimated to be less than $0.5 \mathrm{~s}$. These time uncertainties lead to uncertainties on the estimation of the altitude ranging from 23 to $31 \mathrm{~km}$.

\subsection{Aurorae and magnetic field}

The location of the auroral emission is also very important. It is possible to retrieve the coordinates of the spacecraft and of the tangent point along the line of sight at the time of the detection from the SPICAM data. Fig. 4 represents the projections on the planet of the spacecraft trajectories during orbit 716 and 2800 in dotted lines. The track of the MNP is shown by the black dashed line. Black dots correspond to the times of the auroral detections. These dots can however be separated by several tens of degrees in longitude, which is not accurate enough to determine the actual position of the auroral emissions. To estimate the coordinates of the aurora, we used the $d_{\text {aur }}$ value calculated in Section 2.3, which is the distance between the spacecraft and the aurora, and the distance $D$ from the spacecraft to the MNP, which is given in the data files. The actual estimated locations of the aurorae are thus indicated with white dots. All three occur in the southern hemisphere, where the residual magnetic field is relatively strong. As previously suggested by Leblanc et al. (2008) and Gérard et al. (2015) for nadir observations, these limb detections have been overlaid on a map of probabilities of finding a closed magnetic field line derived from MGS measurements (Brain et al., 2007). It appears that these aurorae are located at the boundaries of open-closed magnetic field
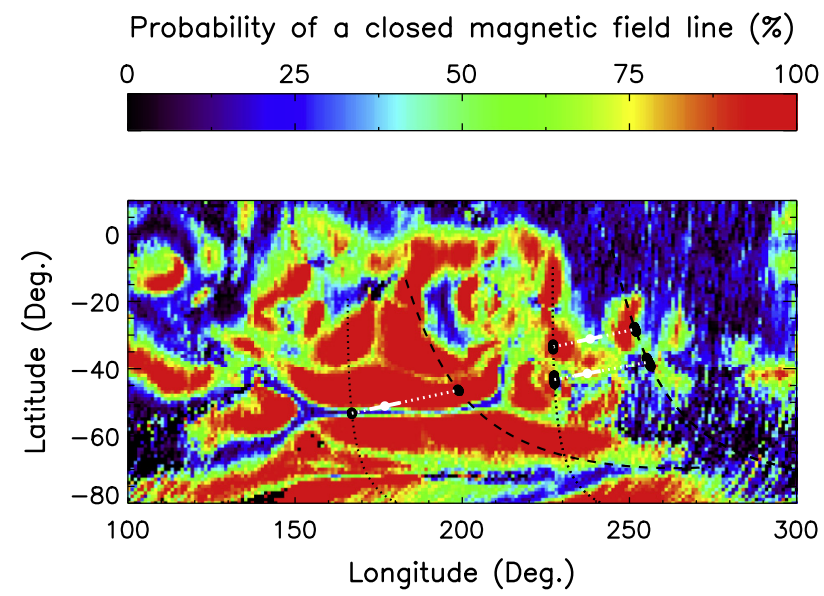

Fig. 4. Location of the auroral ultraviolet emission. The auroral detections are overplotted on a map of the probability to find a closed magnetic field line. The black dotted lines correspond to the spacecraft trace while the dashed lines refer to the trace of the tangent point of the limb observations. The black dots correspond to the time of the detections. The white dots indicate the actual estimated location on the aurorae. Error bars around these dots show the uncertainty of the auroral emission location (see text). 
lines in cusp-like structures, as previously observed for nadir detections. Gérard et al. (2015) suggested that these results indicate that electrons are accelerated by a transient parallel electric field along semi open magnetic field lines. These auroral detections remain in cusp-like areas even when considering an uncertainty of $2 \mathrm{~s}$ in the determination of the peak delay (see error bars in Fig. 4).

\subsection{Other auroral emissions}

So far, we have analyzed the strongest auroral emission, that of the CO $\left(a^{3} \Pi-X^{1} \Sigma\right)$ Cameron bands between 190 and $270 \mathrm{~nm}$. Other emissions can also be observed in the auroral spectra: the $\mathrm{CO}_{2}^{+}$ $\left(\mathrm{B}^{2} \Sigma_{\mathrm{u}}^{+}-\mathrm{X}^{2} \Pi_{\mathrm{g}}\right)$ doublet at $289 \mathrm{~nm}$, the $\mathrm{CO}\left(\mathrm{A}^{1} \Pi-\mathrm{X}^{1} \Sigma^{+}\right) 4 \mathrm{P}$ bands between 135 and $170 \mathrm{~nm}$, and OI multiplets at 130.4 and $297.2 \mathrm{~nm}$. The $\mathrm{CO}_{2}^{+}$doublet emission, peaking at $289 \mathrm{~nm}$, was processed between 286 and $293 \mathrm{~nm}$ as explained for the CO Cameron bands in Section 2.2. This emission is weaker than the CO Cameron bands though, and it has not been possible to detect the emission in bin 5 of Detection III (Table 2). The mean $\mathrm{CO}_{2}^{+}$doublet emission intensity at the maximum is $640 \mathrm{R}$ for Detection I, $455 \mathrm{R}$ for Detection II and $410 \mathrm{R}$ for Detection III. Leblanc et al. (2006a) derived a mean value over the five spatial bins of $2050 \mathrm{R}$ for the CO Cameron bands emission and $205 \mathrm{R}$ for the $\mathrm{CO}_{2}^{+}$doublet emission of Detection I. For Detection II, Leblanc et al. (2008) found averaged intensities of $712 \mathrm{R}$ and $100 \mathrm{R}$ for the $\mathrm{CO}$ and $\mathrm{CO}_{2}^{+}$doublet emissions, respectively. In their nadir study, Leblanc et al. (2008) found a mean value of $58 \mathrm{R}$ for $\mathrm{CO}_{2}^{+}$doublet and Gérard et al. (2015) found a CO Cameron/ $\mathrm{CO}_{2}^{+}$doublet ratio ranging from 2.1 to 7.8 , in good agreement with our limb measurements (Table 2). Our average ratio of 6.1 is also close to the value of $\sim 5.5$ observed in the dayglow at the altitude of the emission peak (Leblanc et al., 2006b).

In this study, the $\mathrm{CO}\left(\mathrm{A}^{1} \Pi-\mathrm{X}^{1} \Sigma^{+}\right)$Four Positive group was also analyzed between 135 and $170 \mathrm{~nm}$. Intensities are given in Table 2, except for the high spectral resolution bins in orbit 2800. They vary from 184 to $1406 \mathrm{R}$ and the CO Cameron/CO 4P ratio ranges between 3.5 and 7.4, with a mean value of 5.1. The oxygen emission at $297.2 \mathrm{~nm}$ is quite variable, with intensities ranging from $98 \mathrm{R}$ to $777 \mathrm{R}$ and CO Cameron/O (297.2 nm) ratios ranging from 1.8 to 16.1 . The oxygen triplet at 130.4 is quite weak. It has not been previously observed in Mars auroral spectra. In this study, we detect the $130.4 \mathrm{~nm}$ OI emission in some spatial bins, either directly in the SPICAM spectra or in the time variations of the signal (such as Fig. 1) between 129 and $131 \mathrm{~nm}$. The mean retrieved intensity for this emission is $31 \mathrm{R}$ and the ratio with the $\mathrm{CO}$ Cameron bands varies from 30.4 to 173 . Finally, we looked for the $135.6 \mathrm{~nm}$ OI multiplet but never detected it. Fox and Stewart (1991) estimated a $130.4 \mathrm{~nm} / 135.6 \mathrm{~nm}$ ratio of $5.5 \pm 2.7$ in nadir observations of the Venus aurora with the PV-OUVS spectrometer.
Applying the same ratio here, we expect the $135.6 \mathrm{~nm}$ oxygen emission to range between 3 and $10 \mathrm{R}$. To estimate the SPICAM sensitivity threshold, we evaluated the 1-sigma background signal variability around this wavelength along time and determined a value of $7.7 \mathrm{R}$. Considering that the $135.6 \mathrm{~nm}$ emission should be at least twice this value to be unambiguously detected, we set an upper limit of this intensity of $\sim 15 \mathrm{R}$, a value that is compatible with the modeled brightness and explains the lack of detection in the aurora with SPICAM.

\section{Electron transport modeling}

In order to understand the excitation processes leading to these auroral emissions, the SPICAM detections will now be compared with simulations made with a model of electron transport in the Martian thermosphere based on a Monte-Carlo method. This model was described by Shematovich et al. (2008) for the Mars dayglow and applied by Gérard et al. (2008) to the Venus dayglow and aurora. It is briefly described in the next section and results obtained for mono-energetic electrons used as input parameters will be described in Section 3.2. Simulations using electron fluxes measured by ASPERA-3 will be discussed in Section 3.3.

\subsection{The Monte Carlo electron transport model}

The Monte Carlo model was initially developed to simulate the production of photoelectrons and their energy degradation in the Martian atmosphere. The model is based on photochemical processes, kinetics and a Monte Carlo approach. Energetic electrons interact with the Martian atmosphere where they can lose their kinetic energy in elastic, inelastic and ionizing collisions with the ambient atmospheric gas. The energy loss of the precipitating electrons is calculated by solving the kinetic Boltzmann equation, involving the transport of electrons, the production rates of primary and secondary electrons and elastic and inelastic scattering terms. The Monte Carlo algorithm is efficient to numerically solve kinetic equations for atmospheric systems in the stochastic approach. It calculates the system evolution from the initial to the steady state between altitudes of $250 \mathrm{~km}$ and $75 \mathrm{~km}$. The outputs of the model are calculated vertical emission profiles for various $\mathrm{CO}, \mathrm{CO}_{2}^{+}$and $\mathrm{O}$ emissions. More details about the Monte-Carlo model and its implementation can be found in Shematovich et al. (1994, 2008).

The neutral atmosphere is taken from outputs of the Mars Global Ionosphere-Thermosphere Model (M-GITM) (Bougher et al., 2015) for a solar longitude of $0^{\circ}$, a latitude of $50^{\circ}$ South, a longitude of $180^{\circ}$ (which correspond to a region with significant residual magnetic field on Mars), a $F 10.7 \mathrm{~cm}$ solar flux of

Table 2

Limb intensities of the auroral emissions.

\begin{tabular}{|c|c|c|c|c|c|c|c|c|c|c|c|}
\hline Orbit & $\begin{array}{l}\text { Spatial } \\
\text { bin }\end{array}$ & Time (s) & $\begin{array}{l}\text { CO intensity } \\
\text { (R) }\end{array}$ & $\mathrm{CO}_{2}^{+}(\mathrm{R})$ & $\begin{array}{l}\mathrm{CO} / \mathrm{CO}_{2}^{+} \\
\text {ratio }\end{array}$ & $\mathrm{CO} 4 \mathrm{P}(\mathrm{R})$ & $\begin{array}{l}\mathrm{CO} / \mathrm{CO} 4 \mathrm{P} \\
\text { ratio }\end{array}$ & OI $297.2 \mathrm{~nm}(\mathrm{R})$ & $\begin{array}{l}\mathrm{CO} / \mathrm{OI} \\
\text { ratio }\end{array}$ & OI $130.4 \mathrm{~nm}(\mathrm{R})$ & $\begin{array}{l}\mathrm{CO} / \mathrm{OI} \\
\text { ratio }\end{array}$ \\
\hline \multirow[t]{4}{*}{ 716A01 } & 1 & $535-540$ & 4959 & 759 & 6.5 & 1406 & 3.5 & 718 & 6.9 & 53 & 94.3 \\
\hline & 2 & $532-540$ & 4871 & 1096 & 4.4 & 1236 & 3.9 & - & - & 29 & 166.8 \\
\hline & 4 & $532-538$ & 4462 & 272 & 16.4 & 923 & 4.8 & 306 & 14.6 & 45 & 98.6 \\
\hline & 5 & $531-538$ & 4043 & 420 & 9.6 & 587 & 6.9 & - & - & 23 & 173.0 \\
\hline \multirow[t]{8}{*}{ 2800A02 } & 1 & $370-395$ & 1338 & 471 & 2.8 & - & - & 718 & 1.8 & - & - \\
\hline & 2 & $370-395$ & 2371 & 553 & 4.3 & - & - & 777 & 3.0 & - & - \\
\hline & 4 & $373-392$ & 2216 & 372 & 6.0 & 298 & 7.4 & 171 & 13.0 & 23 & 97.9 \\
\hline & 5 & $373-393$ & 2396 & 269 & 8.9 & 364 & 6.6 & 149 & 16.1 & 17 & 142.7 \\
\hline & 1 & $515-550$ & 1008 & 471 & 2.1 & - & - & 255 & 4.0 & - & - \\
\hline & 2 & 509-519 & 1211 & 578 & 2.1 & - & - & 287 & 4.2 & - & - \\
\hline & 4 & $515-550$ & 694 & 169 & 4.1 & 184 & 3.8 & 98 & 7.1 & - & - \\
\hline & 5 & $515-550$ & 733 & - & - & 184 & 4.0 & 125 & 5.9 & 24 & 30.4 \\
\hline
\end{tabular}


$30 \times 10^{-22} \mathrm{~W} \mathrm{~m}^{-2} \mathrm{~Hz}^{-1}$ at Mars (average value at the time of the auroral detections) and at midnight local time. The M-GITM takes into account the fundamental physical parameters, ion-neutral chemistry, and key radiative processes of Mars from the ground to the exosphere $(0-250 \mathrm{~km})$. The processes that govern the relative importance of the collisional processes and energy loss of the electrons were listed in Shematovich et al. (2008). The cross sections for electron impact dissociative excitation and ionization of the $\mathrm{CO}_{2}$ states and their analytical expression are taken from the compilation by Shirai et al. (2001). The excitation function of the $\mathrm{CO}\left(\mathrm{a}^{3} \Pi\right)$ state by electron impact on $\mathrm{CO}_{2}$ is well known but the absolute normalization is still largely uncertain. It was initially measured by Ajello (1971) and was re-evaluated several times (Erdman and Zipf, 1983; Avakyan et al., 1998). It was recently corrected to account for the determination of the $\mathrm{CO}\left(\mathrm{a}^{3} \Pi\right)$ radiative lifetime (Gilijamse et al., 2007; Bhardwaj and Jain, 2013) and we have adopted a value of $8 \times 10^{-17} \mathrm{~cm}^{2}$ at $80 \mathrm{eV}$ in this study (Gronoff et al., 2012). A second source of CO $\left(a^{3} \Pi\right)$ state is the direct excitation by electron impact on $\mathrm{CO}$. The corresponding excitation cross section is taken from Shirai et al. (2001), as well as for the $\mathrm{CO}\left(\mathrm{A}^{2} \Pi\right)$ state leading to the Fourth Positive bands. The cross section for the excitation of the $\mathrm{O}\left({ }^{3} \mathrm{~S}^{\circ}\right)$ and $\mathrm{O}\left({ }^{5} \mathrm{~S}\right)$ by electron impact on $\mathrm{O}$ atoms corresponding to the emissions at 130.4 and $135.6 \mathrm{~nm}$ are taken from Itikawa and Ichimura (1990).

The Monte Carlo model of electron transport rests upon several hypotheses. First, it is assumed that the ambient atmospheric gas is characterized by the local Maxwellian velocity distribution function. Second, no magnetic field is considered in this model. Finally, the electrons are assumed to precipitate perpendicularly to the atmosphere of the planet. These hypotheses might not reflect reality though, as Gérard et al. (2015) showed that electrons appear to accelerate in cusp-like regions. They also showed that aurorae can occur under conditions where the magnetic field lines are inclined by an angle ranging from $83^{\circ}$ (B field pointing downward nearly vertically) to $0.2^{\circ}$ (quasi horizontal), based on the statistical map of the inclination angle by Connerney et al. (2001).

\subsection{Mono-energetic electrons}

First, the Monte Carlo model is run with mono-energetic electrons with an isotropic pitch-angle distribution in the downward direction at the top of the model. The energy flux is fixed to $1 \mathrm{~mW} \mathrm{~m}^{-2}$ and simulations are made for electron energies ranging from $E_{0}=50$ to $1000 \mathrm{eV}$. These boundaries have been chosen since: (i) below $\sim 20 \mathrm{eV}$, cross sections for electron impact on $\mathrm{CO}_{2}$ are small. They fall below $10 \%$ of their peak value at $20 \mathrm{eV}$ for the $\mathrm{e}+\mathrm{CO}_{2} \rightarrow \mathrm{CO}_{2}^{+}\left(\mathrm{B}^{2} \Sigma\right)+2 \mathrm{e}, 13 \mathrm{eV}$ for the $\mathrm{e}+\mathrm{CO}_{2} \rightarrow \mathrm{CO}\left(\mathrm{a}^{3} \Pi\right)+\mathrm{e}$ and $6 \mathrm{eV}$ for the $\mathrm{e}+\mathrm{CO} \rightarrow \mathrm{CO}\left(\mathrm{a}^{3} \Pi\right)+$ e processes; (ii) in situ measurements made by Brain et al. (2006) and Lundin et al. (2006) showed that the highest energies in these discrete auroral events do not exceed $1000 \mathrm{eV}$. The vertical emission profiles calculated by the model for the CO Cameron bands at several initial electron energies are shown with colored lines in Fig. 5a. The maximum peak volume emission rate is obtained for $E_{0} \approx 500 \mathrm{eV}$. The altitude of the peak emission increases with decreasing electron energy (Fig. 5b), ranging from 116 to $141 \mathrm{~km}$. The values $E_{0}=50 \mathrm{eV}$ and $1000 \mathrm{eV}$ being considered as the lower and upper limits, these altitudes can also be taken as upper and lower boundaries of Martian auroral emissions. These values are in good agreement with our estimated altitudes deduced from the observations. Detection I $(132 \mathrm{~km})$, Detection II $(143 \mathrm{~km})$ and Detection III $(137 \mathrm{~km})$ would correspond to electrons with energies of respectively $\sim 190 \mathrm{eV}$, $40 \mathrm{eV}$ and $90 \mathrm{eV}$, if they were mono-energetic.

The Monte Carlo model independently calculates the production of the $\mathrm{CO}$ Cameron bands from the $\mathrm{CO}_{2}$ dissociative excitation
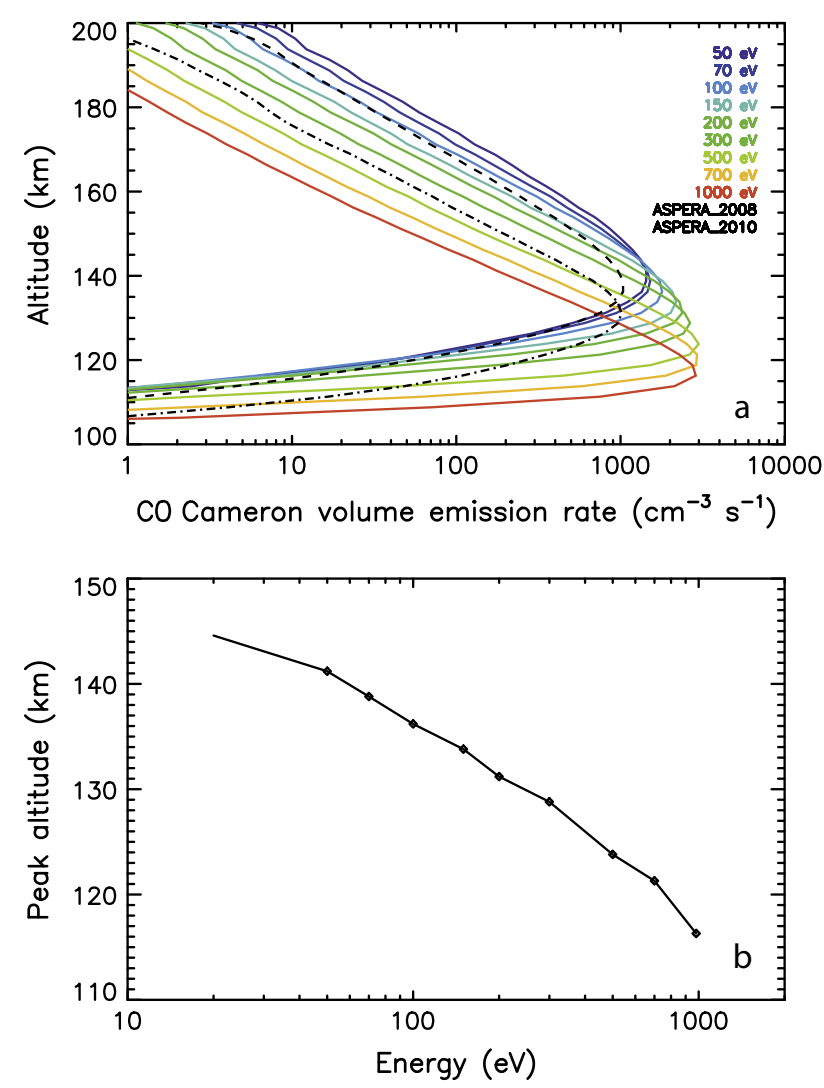

Fig. 5. (a) Monte Carlo auroral model simulations of the CO Cameron volume emission rate for different initial electron energy spectra. The solid colored lines correspond to mono-energetic fluxes as input parameters of the model while the dashed lines correspond to ASPERA-3/ELS electron energy spectra. (b) The peak altitude decreases with increasing initial energies.

and from direct $\mathrm{CO}$ excitation processes. Table 3 shows that $\mathrm{CO}_{2}$ dissociation prevails but that the ratio between the two processes varies with $E_{0}$. In all simulations, the direct excitation from electron impact on $\mathrm{CO}$ contributes a significant fraction to the total Cameron bands intensity. Model results also indicate that the $\mathrm{CO}$ Cameron $/ \mathrm{CO}_{2}^{+}$intensity ratio increases with decreasing energies, with values ranging from 2.5 to 8 , in relatively good agreement with half the auroral observations (see Table 2). These values are lower than those estimated from the other half of the observations in Section 2.5. This possibly stems from the fact that auroral electron spectra are distributed over a range of energies rather than mono-energetic. In particular, a low energy electron component is present in the observed electron energy spectra, which increases the $\mathrm{CO}$ Cameron $/ \mathrm{CO}_{2}^{+}$ratio. Due to the uncertainties on the cross sections for the production of these emissions, we made some sensitivity tests. As expected, doubling the cross section for the production of $\mathrm{CO}_{2}^{+}$leads to an increase of its intensity by a factor of two. Doubling the cross section of the production of CO Cameron from $\mathrm{CO}_{2}$ dissociative excitation increases the calculated total $\mathrm{CO}$ Cameron bands intensity by $70-80 \%$ and doubling the cross section of the production of $\mathrm{CO}$ Cameron from $\mathrm{CO}$ dissociative excitation increases the calculated total CO Cameron bands intensity by 19$28 \%$.

The Monte-Carlo model also simulates the $135.6 \mathrm{~nm}$ oxygen emission, which, up to present, has not been observed in the Mars atmosphere. The ratio between the 130.4 and the $135.6 \mathrm{~nm}$ emissions is found to be $\sim 2.8$. However, Gérard et al. (2008) showed that this ratio increases by a factor of $\sim 3$ when considering multiple scattering of the $130.4 \mathrm{~nm}$ multiplet in the optically thick 
Table 3

Monte-Carlo vertical intensity simulations for mono-energetic auroral electrons.

\begin{tabular}{|c|c|c|c|c|c|c|c|}
\hline$E_{0}(\mathrm{eV})$ & Energy flux $\left(\mathrm{mW} \mathrm{m}^{-2}\right)$ & $\mathrm{CO}$ Cameron (from $\left.\mathrm{CO}_{2}\right)(\mathrm{R})$ & CO Cameron (from CO) (R) & $\mathrm{CO}_{2}^{+}(\mathrm{R})$ & $\mathrm{CO} 4 \mathrm{P}(\mathrm{R})$ & OI $130.4(\mathrm{R})$ & OI $135.6(\mathrm{R})$ \\
\hline 50 & 1.00 & 2700 & 980 & 490 & 68 & 510 & 170 \\
\hline 100 & 1.00 & 3100 & 880 & 850 & 69 & 340 & 110 \\
\hline 200 & 1.00 & 3500 & 930 & 1200 & 76 & 250 & 86 \\
\hline 500 & 1.00 & 3800 & 930 & 1500 & 80 & 150 & 57 \\
\hline 1000 & 1.00 & 3500 & 840 & 1700 & 76 & 100 & 40 \\
\hline ASPERA-2008 & 0.84 & 1300 & 500 & 450 & 30 & 99 & 37 \\
\hline ASPERA-2010 & 1.72 & 3200 & 1250 & 765 & 79 & 520 & 190 \\
\hline
\end{tabular}

a See Fig. 5a and text.

Venus atmosphere. Assuming a similar enhancement in the Mars atmosphere, the modeled ratio would be as high as 8.4, explaining why the $135.6 \mathrm{~nm}$ OI emission cannot be observed at the SPICAM sensitivity level (see Section 2.5).

\subsection{ASPERA electron energy spectra}

The Monte Carlo model described in Gérard et al. (2008) has been applied to Mars and improved for this study. In particular, the input electron spectra can have any analytical or measured energy distribution. In this case, these spectra have been recorded with the Electron Spectrometer (ELS) of the ASPERA-3 instrument on board Mars Express (Barabash et al., 2006). It provides electron energy spectra measurements every $4 \mathrm{~s}$ between $10 \mathrm{eV}$ and $20 \mathrm{keV}$ with $8 \%$ energy resolution. Examples of ASPERA-3/ELS electron energy spectra have been presented by Lundin et al. (2006). Some of these spectra have also been described by Gérard et al. (2015) as they were concurrent in time with nadir auroral observations. For comparison, we use two ASPERA-3/ELS electron energy spectra acquired on 7 September 2008 at $38^{\circ} \mathrm{S}$ and $171^{\circ}$ of longitude and on 10 May 2010 at $17^{\circ} \mathrm{S}$ and $193^{\circ}$ of longitude. Their respective characteristics are a total downward energy flux of 0.84 and $1.72 \mathrm{~mW} \mathrm{~m}^{-2}$, peak energies of 402 and $144 \mathrm{eV}$, and average electron energies of 380 and $140 \mathrm{eV}$. Intensities calculated with these input spectra are reported in Table 3. The calculated CO Cameron volume emission rates are shown in Fig. 5a as black dashed-dotted and dashed lines, respectively. They closely resemble the emission profiles previously obtained for mono-energetic electrons. They are in good agreement with the volume emission rates corresponding to the mono-energetic simulations for energy values $E_{0}$ close to the peak energy determined from the shape of ASPERA spectra. Despite the fact that the precipitating electron energy fluxes are not equal to $1 \mathrm{~mW} \mathrm{~m}^{-2}$ as in the mono-energetic electron simulations, the volume emission rates predicted for the ASPERA-3/ELS electron fluxes appear to be smaller than those calculated in Section 3.2. This is probably because the ASPERA spectra also contain a low electron energy component.

\subsection{ASPERA electron energy spectra for nadir SPICAM observations}

Gérard et al. (2015) showed that each of their nadir auroral detection can be associated with an increase in the ASPERA-3/ELS measurement of the electron energy flux. Here, these electron energy spectra have been used as input parameters of the Monte-Carlo model to simulate nadir auroral intensities. These simulated intensities are here compared to those observed by Gérard et al. (2015). Results are shown in Table 4. Predicted intensities are higher than the observed values. However, Gérard et al. (2015) pointed out that the precipitated flux could follow magnetic field lines inclined by several tens of degrees, based on the Mars Global Surveyor measurements of the magnetic field components (Connerney et al., 2001) and the time delay observed between the precipitated flux of electron by ASPERA and the nadir auroral emission detection by SPICAM. By contrast, the model only considers downward fluxes perpendicular to the atmosphere and integrates the volume emission rate over the full vertical direction. This different geometry between the nadir observations of the inclined and confined field aligned aurora and the model simulations possibly explains the overestimated modeled intensities. Also, part of the electrons mirror above the region of auroral emission, a process that is not considered by the model where all electrons contribute to the auroral process. The lack of consistency between the observed intensities and the simulated intensities using observed electron energy spectra and measured precipitating fluxes of electron appears to confirm the lack of correlation between downgoing energy fluxes and auroral emissions (Leblanc et al., 2008; Gérard et al., 2015).

\section{Conclusions}

The entire database of SPICAM-UV limb observations made in the Southern hemisphere during nighttime has been analyzed and the number of limb auroral detections is now extended to three events, lasting a few seconds each. Once again, it was shown that auroral emissions occur at the boundary of open-closed magnetic field lines, in agreement with earlier studies based on nadir observations. The CO $\left(\mathrm{a}^{3} \Pi-\mathrm{X}^{1} \Sigma\right)$ Cameron bands, the CO $\left(\mathrm{A}^{1} \Pi-\right.$ $\left.\mathrm{X}^{1} \Sigma^{+}\right)$Four Positive system, the $\mathrm{CO}_{2}^{+}\left(\mathrm{B}^{2} \Sigma_{\mathrm{u}}^{+}-\mathrm{X}^{2} \Pi_{\mathrm{g}}\right)$ doublet, the OI multiplets at $297.2 \mathrm{~nm}$ and at $130.4 \mathrm{~nm}$ were detected in the limb spectra and their intensities have been quantified. The mean intensities deduced for these emissions reach $2525 \mathrm{R}, 648 \mathrm{R}, 494 \mathrm{R}$, $360 \mathrm{R}$ and $31 \mathrm{R}$, respectively. The variability is very important. The $135.6 \mathrm{~nm}$ OI emission was not detected, most likely because its brightness falls below the SPICAM sensitivity threshold. The altitude of the CO Cameron bands auroral emissions was found to be $137 \pm 27 \mathrm{~km}$, in very good agreement with Monte-Carlo simulations using, for the first time, APERA-3/ELS electron energy distributions as input parameters. The model also suggests that mono-energetic electrons ranging from 50 to $1000 \mathrm{eV}$, which are typical values measured by in situ electron spectrometers, should lead to auroral emissions occurring between 116 and $141 \mathrm{~km}$. Krymskii et al. (2002) indicated that, in the presence of numerous cusp-like regions above the crustal anomalies through which charged particles can penetrate to the lower layers of the neutral atmosphere, significant heating leading to temperature increases may be expected. We note however that Stiepen et al. (2015a) could not find any correlation between the crustal field configuration and the dayside exospheric temperature derived from the airglow topside scale height.

Despite the new information obtained in this study, the analysis could only be carried out for three auroral cases. This thus confirms that Martian auroral emissions are very rare events at the level of the SPICAM-UV instrument sensitivity. A larger number of auroral events was detected by SPICAM-UV in the nadir mode though 
Table 4

Auroral nadir intensities ${ }^{\mathrm{a}}$ and Monte-Carlo simulations.

\begin{tabular}{|c|c|c|c|c|c|c|c|c|}
\hline \multirow[t]{2}{*}{ Date } & \multirow[t]{2}{*}{$\begin{array}{l}\text { Satellite } \\
\text { altitude (km) }\end{array}$} & \multirow[t]{2}{*}{$\bar{E}(\mathrm{eV})$} & \multicolumn{2}{|c|}{$\begin{array}{l}\text { CO Cameron bands nadir } \\
\text { intensity }(\mathrm{R})\end{array}$} & \multirow{2}{*}{$\begin{array}{l}\text { CO Cameron } \\
\text { bands simulated } \\
\text { intensity }(R)\end{array}$} & \multicolumn{2}{|c|}{$\mathrm{CO}_{2}^{+}$nadir intensity $(\mathrm{R})$} & \multirow[t]{2}{*}{$\begin{array}{l}\mathrm{CO}_{2}^{+} \text {simulated } \\
\text { intensity }(\mathrm{R})\end{array}$} \\
\hline & & & $\begin{array}{l}\text { Spatial bins } \\
1 \text { and } 2\end{array}$ & $\begin{array}{l}\text { Spatial bins } \\
4 \text { and } 5\end{array}$ & & $\begin{array}{l}\text { Spatial bins } \\
1 \text { and } 2\end{array}$ & $\begin{array}{l}\text { Spatial bins } \\
4 \text { and } 5\end{array}$ & \\
\hline \multirow[t]{2}{*}{ 2004-07-07 } & 737 & 287 & 730 & 210 & 5685 & 240 & 50 & 1329 \\
\hline & 691 & 228 & 770 & 590 & 4072 & 920 & 110 & 864 \\
\hline $2005-12-27$ & 917 & 224 & - & 440 & 3459 & - & 175 & 753 \\
\hline $2006-02-17$ & 342 & 330 & 870 & 320 & 2602 & - & 100 & 577 \\
\hline \multirow[t]{2}{*}{ 2006-02-19 } & 396 & 340 & 1510 & 1180 & 438 & 460 & 190 & 91 \\
\hline & 321 & 150 & 1010 & 735 & 1357 & 155 & 95 & 237 \\
\hline 2008-07-07 & 2961 & 270 & 660 & 495 & 309 & 380 & 220 & 67 \\
\hline \multirow[t]{2}{*}{ 2008-09-07 } & 1356 & 380 & - & 295 & 1821 & - & - & 451 \\
\hline & 1259 & 207 & 2375 & 1880 & 1241 & - & - & 274 \\
\hline 2010-05-10 & 591 & 140 & 525 & 870 & 4462 & - & 205 & 765 \\
\hline \multirow[t]{2}{*}{$2010-10-15$} & 817 & 325 & \multirow{2}{*}{1590} & \multirow{2}{*}{795} & 17,983 & \multirow{2}{*}{-} & \multirow{2}{*}{380} & 4546 \\
\hline & 793 & 380 & & & 11,308 & & & 2847 \\
\hline
\end{tabular}

a From Gérard et al. (2015).

(Gérard et al., 2015). This may appear unexpected at first since limb observations increase emission brightness by integrating the intensity along the line of sight. The integrated intensity at the limb may thus become larger than the detection threshold of the instrument and allow detection of emission too weak to be observable at the nadir. For example, Mars NO UV nightglow emissions cannot be detected at the nadir with the SPICAM sensitivity but can easily be observed at the limb (Stiepen et al., 2015b). For the auroral emissions, intensities retrieved at the limb and at the nadir are comparable though. It indicates that these discrete southern aurorae are horizontally very confined compared to the emitting layer of airglows. Therefore, Mars aurorae are bright enough to be detected over the NO nightglow in nadir observations but not in the limb geometry, where the entire NO layer is integrated and overlaps the very confined auroral emissions. Thus, many other auroral events probably exist in the SPICAM-UV limb database, but they are obscured by the NO nightglow emission in the same wavelength region.

\section{Acknowledgments}

We gratefully thank all members of the ESA Mars Express project and of the SPICAM and ASPERA-3 scientific and technical teams. We also thank $\mathrm{S}$. Bougher for providing the neutral atmosphere modeled by its M-GITM and R. Lundin for the ASPERA-3/ ELS electron energy spectra used for the Monte-Carlo simulations. This research was supported by the PRODEX program managed by the European Space Agency with the help of the Belgian Federal Space science Policy Office. This work was also funded by the Centre National d'Etudes Spatiales. The Mars Express data used in this study are available on the ESA's Planetary Data Archive web site http://www.sciops.esa.int/index.php?project=PSA\&page= mex. Finally, we would like to thank both reviewers for their useful comments on the manuscript.

\section{References}

Ajello, J.M., 1971. Emission cross sections of $\mathrm{CO}_{2}$ by electron impact in the interval 1260-4500 Å. II. J. Chem. Phys. 55, 3169-3177. http://dx.doi.org/10.1063/ 1.1676564 .

Avakyan, S.V. et al., 1998. Collision Processes and Excitation of Ultraviolet Emission from Planetary Atmospheric Gases: A Handbook of Cross Sections. Gordon and Breach, London.

Barabash, S. et al., 2006. The analyzer of space plasmas and energetic atoms (ASPERA-3) for the Mars Express mission. Space Sci. Rev. 126, 113-164. http:/ dx.doi.org/10.1007/s11214-006-9124-8.

Bertaux, J.-L. et al., 2005a. Discovery of an aurora on Mars. Nature 435, 790-794. http://dx.doi.org/10.1038/nature03603.
Bertaux, J.-L. et al., 2005b. Nightglow in the upper atmosphere of Mars and implications for atmospheric transport. Science 307, 566-569. http://dx.doi. org/10.1126/science.1106957.

Bertaux, J.-L. et al., 2006. SPICAM on Mars Express: Observing modes and overview of UV spectrometer data and scientific results. J. Geophys. Res. 111, E10S90. http://dx.doi.org/10.1029/2006JE002690.

Bhardwaj, A., Jain, S.K., 2013. CO Cameron band and $\mathrm{CO}_{2}^{+}$UV doublet emissions in the dayglow of Venus: Role of $\mathrm{CO}$ in the Cameron band production. J. Geophys. Res. 118, 3660-3671. http://dx.doi.org/10.1002/jgra.50345.

Bougher, S.W.D. et al., 2015. Mars Global Ionosphere-Thermosphere Model (MGITM): Solar cycle, seasonal, and diurnal variations of the Mars upper atmosphere. J. Geophys. Res.: Planets 120. http://dx.doi.org/10.1002/ 2014JE004715.

Brain, D.A. et al., 2006. On the origin of aurorae on Mars. Geophys. Res. Lett. 33, L01201. http://dx.doi.org/10.1029/2005GL024782.

Brain, D.A. et al., 2007. Electron pitch angle distributions as indicators of magnetic field topology near Mars. J. Geophys. Res. 112, A09201. http://dx.doi.org/ 10.1029/2007JA012435.

Connerney, J.E.P. et al., 2001. The global magnetic field of Mars and implications for crustal evolution. Geophys. Res. Lett. 28, 4015-4018. http://dx.doi.org/10.1029/ 2001GL013619.

Cox, C. et al., 2008. Distribution of the ultraviolet nitric oxide martian night airglow: Observations from Mars Express and comparisons with a one-dimensional model. J. Geophys. Res. 113, E08012. http://dx.doi.org/10.1029/2007JE003037.

Erdman, P.W., Zipf, E.C., 1983. Electron-impact excitation of the Cameron system (a3P yields X1S) transition of CO. Planet. Space Sci. 31, 317-321. http://dx.doi. org/10.1029/JA090iA11p11087.

Fox, J.L., 1992. Airglow and aurora in the atmospheres of Venus and Mars. In: Luhmann, J.G., Tatrallyay, M., Pepin, R.O. (Eds.), Venus and Mars: Atmospheres, Ionospheres, and Solar Wind Interactions. American Geophysical Union, pp. 191-222, http://dx.doi.org/10.1029/GM066p0191.

Fox, J.L., Stewart, A.I.F., 1991. The Venus ultraviolet aurora: A soft electron source. J. Geophys. Res. 96, 9821-9828. http://dx.doi.org/10.1029/91JA00252.

Gérard, J.-C. et al., 2008. The Venus ultraviolet oxygen dayglow and aurora: Model comparison with observations. Planet. Space Sci. 56, 542-552. http://dx.doi.org/ 10.1016/j.pss.2007.11.008.

Gérard, J.-C. et al., 2015. Concurrent observations of ultraviolet aurora and energetic electron precipitation with Mars Express. J. Geophys. Res. 120. http://dx.doi.org/ 10.1002/2015JA021150.

Gilijamse, J.J. et al., 2007. The radiative lifetime of metastable CO (a3P, $n=0$ ). J. Chem. Phys. 127 (22), 221102-1-221102-4. http://dx.doi.org/10.1063/ 1.2813888.

Gronoff, G. et al., 2012. Computing uncertainties in ionosphere-airglow models: II. The martian airglow. J. Geophys. Res. 117, A05309. http://dx.doi.org/10.1029/ 2011JA017308.

Itikawa, Y., Ichimura, A., 1990. Cross sections for collisions of electrons and photons with atomic oxygen. J. Phys. Chem. Ref. Data 19, 637. http://dx.doi.org/10.1063/ 1.555857.

Krymskii, A.M. et al., 2002. Structure of the magnetic field fluxes connected with crustal magnetization and topside ionosphere at Mars. J. Geophys. Res. 107 (A9), 1245. http://dx.doi.org/10.1029/2001JA000239.

Leblanc, F. et al., 2006a. Origins of the martian aurora observed by Spectroscopy for Investigation of Characteristics of the Atmosphere of Mars (SPICAM) on board Mars Express. J. Geophys. Res. 111, A09313. http://dx.doi.org/10.029/ 2006JA011763.

Leblanc, F. et al., 2006b. Martian dayglow as seen by the SPICAM UV spectrograph on Mars Express. J. Geophys. Res. 111, E09S11. http://dx.doi.org/10.1029/ 2005JE002664.

Leblanc, F. et al., 2008. Observations of aurorae by SPICAM ultraviolet spectrograph on board Mars Express: Simultaneous ASPERA-3 and MARSIS measurements. J. Geophys. Res. 113, A08311. http://dx.doi.org/10.1029/2008JA013033. 
Lundin, R. et al., 2006. Plasma acceleration above martian magnetic anomalies. Science 311, 980-983. http://dx.doi.org/10.1126/science.1122152.

Shematovich, V.I., Bisikalo, D.V., Gérard, J.C., 1994. A kinetic model of the formation of the hot oxygen geocorona: 1. Quiet geomagnetic conditions. J. Geophys. Res. 99 (A12), 23217-23228. http://dx.doi.org/10.1029/94JA01769.

Shematovich, V.I. et al., 2008. Monte Carlo model of electron transport for the calculation of Mars dayglow emissions. J. Geophys. Res. 113 (E2). http://dx.doi. org/10.1029/2007JE002938.
Shirai, T., Tabata, T., Tawara, H., 2001. Analytic cross sections for electron collisions with $\mathrm{CO}, \mathrm{CO}_{2}$, and $\mathrm{H}_{2} \mathrm{O}$ relevant to edge plasma impurities. Atom. Data Nucl. Data Tables 79, 143-184, pii: S0092640X01908666.

Stiepen, A. et al., 2015a. Mars thermospheric scale height: CO Cameron and $\mathrm{CO}_{2}^{+}$ dayglow observations from Mars Express. Icarus 245, 295-305. http://dx.doi. org/10.1016/j.icarus.2014.09.051.

Stiepen, A. et al., 2015b. Ten years of martian nitric oxide nightglow observations. Geophys. Res. Lett. http://dx.doi.org/10.1002/2014GL062300. 